\title{
High indoleamine-2,3-dioxygenase 1 (IDO) activity is linked to primary resistance to immunotherapy in non-small cell lung cancer (NSCLC)
}

\author{
Florian Kocher ${ }^{1}$, Arno Amann ${ }^{1}$, Kai Zimmer ${ }^{1}$, Simon Geisler ${ }^{2}$, Dietmar Fuchs ${ }^{2}$, Renate Pichler ${ }^{3}$, Dominik \\ Wolf $^{1,4}$, Katharina Kurz ${ }^{5}$, Andreas Seeber ${ }^{1 *}$, Andreas Pircher ${ }^{1 *}$ \\ ${ }^{1}$ Department of Internal Medicine V (Hematology and Oncology), Comprehensive Cancer Center Innsbruck, Medical University of Innsbruck, \\ Innsbruck, Austria; ${ }^{2}$ Division of Biological Chemistry, Biocenter, Medical University of Innsbruck, Innsbruck, Austria; ${ }^{3}$ Department of Urology, \\ Medical University of Innsbruck, Innsbruck, Austria; ${ }^{4}$ Medical Clinic III, Oncology, Hematology, Immunoncology and Rheumatology, University \\ Clinic Bonn (UKB), University of Bonn, Bonn, Germany; ${ }^{5}$ Department of Internal Medicine II (Infectious Diseases, Immunology, Pneumology, \\ Rheumatology), Medical University of Innsbruck, Innsbruck, Austria \\ Contributions: (I) Conception and design: F Kocher, A Amann, K Zimmer, A Seeber, A Pircher; (II) Administrative support: S Geisler, R Pichler, K Kurz; \\ (III) Provision of study materials or patients: A Amann, A Seeber, A Pircher; (IV) Collection and assembly of data: F Kocher, K Zimmer, R Pichler; (V) Data \\ analysis and interpretation: D Fuchs, S Geisler, A Amann, K Zimmer; (VI) Manuscript writing: All authors; (VII) Final approval of manuscript: All authors. \\ *These authors contributed equally to this work for the senior authorship. \\ Correspondence to: Andreas Seeber, MD, PhD. Department of Internal Medicine V (Hematology and Oncology), Comprehensive Cancer Center \\ Innsbruck, Anichstrasse 35 - 6020, Innsbruck, Austria. Email: andreas.seeber@tirol-kliniken.at.
}

Background Metabolic profiling in non-small cell lung cancer (NSCLC) may identify key metabolic vulnerabilities and shows enormous discovery potential. Preclinical studies showed that metabolic rewiring in cancer plays an essential role in modulation of immunotherapy response. However, this situation is understudied in the clinical setting. Therefore, we aimed to evaluate the plasma metabolic profile of immune checkpoint inhibitor (CI) responding versus non-responding NSCLC patients. The aim of this project is to identify potential predictive biomarkers for CI response.

Methods: Plasma samples from CI treated NSCLC patients were analysed at baseline and at the first follow up scan by using a broad targeted metabolomics mass spectrometry panel, and were compared to healthy controls. For further validation of identified key alterations high-performance liquid chromatography (HPLC) for tryptophan (Trp) and kynurenine (Kyn) as indicator of IDO-activity was performed.

Results: Sixty-seven metabolites were significantly altered in NSCLC patients compared to healthy controls. The metabolic profile of patients with primary CI resistance showed an increase in indoleamine2,3-dioxygenase (IDO) and a decrease in branched-chain amino acids (BCAA) compared to baseline concentrations. Deregulated IDO activity was validated by additional HPLC measurements, which revealed that baseline Trp levels were predictive for CI response. According to receiver operating characteristic (ROC)-analysis baseline Trp levels $\geq 49.3 \mu \mathrm{mol} / \mathrm{L}$ predicted disease control at the first follow up scan with a sensitivity of $89 \%$ and a specificity of $71 \%$.

Conclusions: We showed that NSCLC patients are characterized by a distinct metabolic profile compared to healthy controls. Moreover, metabolic changes during CI therapy were observed. Of those IDO metabolism seemed to play an important role in primary CI resistance. Trp as a surrogate parameter of IDO activity is a promising biomarker in patients undergoing treatment with CIs and might be a future marker in trials investigating IDO inhibitors.

Koywords: Checkpoint inhibitor (CI); indoleamine-2,3-dioxygenase (IDO); non-small cell lung cancer (NSCLC); tryptophan (Trp); metabolic profile

Submitted Mar 03, 2020. Accepted for publication Sep 30, 2020.

doi: $10.21037 /$ tlcr-20-380

View this article at: http://dx.doi.org/10.21037/tlcr-20-380 


\section{Introduction}

Even though a significant reduction of lung cancer mortality has been observed over the last decade, it still represents the most common cause of cancer related death (1). With the approval of checkpoint inhibitors (CI), new treatment options in non-small cell lung cancer (NSCLC) have emerged (2-4). Even though long-lasting responses and prolongation of overall survival (OS) are proven, only a small subset of patients benefits from CI treatment (5). Currently there is a quest towards the identification of predictive biomarkers for CI.

For a long time it has been considered that tumorigenesis, proliferation, metastatic spread and resistance in cancer is driven solely by genetic alterations. However, in addition to genetic alterations, cancer cells have to undergo adaptive metabolic reprogramming to enable adequate nutritional supply and to counterbalance hypoxic stress of the tumor microenvironment (TME) to ensure their survival (6-13). Thus, it has been acknowledged that metabolic rewiring is an important hallmark in cancer (11). Therefore, systematic analyses of the metabolic profile in cancer patients may help to identify key metabolic vulnerabilities favoring cancer homeostasis (14).

First reports indicate the importance of metabolic adaptations towards resistance mechanisms of CI therapy, but further research is needed to define the role and impact of various metabolic processes. For example, it was reported that mutations in Kelch-like ECHassociated protein 1 (KEAP1) and serine/threonine kinase 11 (STK11) are predictors for primary CI resistance (15). Mechanistically both genes (KEAP1, STK11) are crucial players in signaling pathways that facilitate the adaption to oxidative and metabolic stress, and lead to cancer specific metabolic reprogramming. Furthermore, it was shown that deregulation of the oxidative metabolism is associated with poor outcome in melanoma patients undergoing CI therapy (16).

In the present study we investigated the metabolic profile in NSCLC and compared it to healthy controls. Moreover, we aimed to identify plasma metabolic alterations with a broad mass spectrometry based approach and further validated identified features. In particular, we were primarily interested in the metabolic state of primary CI resistant NSCLC patients.

We present the following article in accordance with the STARD reporting checklist (available at http://dx.doi. org/10.21037/tlcr-20-380).

\section{Methods}

\section{Study design and patients}

Serum and plasma of metastatic NSCLC patients who were treated with a CI (either nivolumab or pembrolizumab) as a second-line treatment at the Medical University of Innsbruck were prospectively included from March 2016 until August 2017. All patients received follow-up scans every twelve weeks or earlier in case of clinical suspicion of tumor progression. A cohort of 20 healthy individuals served as control group to delineate metabolic differences from lung cancer patients.

The study has conformed to the provisions of the Declaration of Helsinki (as revised in 2013).

The study was approved by the local ethic committee of the Medical University of Innsbruck (local ethics committee study number: AN2015-0017 345/4.20) and informed consent was taken from all the patients.

\section{Metabolite analysis}

A targeted metabolomics approach with the AbsoluteIDQ ${ }^{\mathrm{TM}}$ p180 kit (BIOCRATES Life Sciences AG, Innsbruck, Austria) was used for quantification, based on electrospray ionization liquid (ESI-LC-MS/MS) and flow-injection analysis mass spectrometry (FIA/MS) measurements. The assay allows simultaneous quantification of in total 167 metabolites out of $10 \mu \mathrm{L}$ peripheral blood plasma, including amino acids, biogenic amines, acylcarnitines, glycerophospholipids, sphingomyelins, and the sum of hexoses.

\section{Tryptophan (Trp) and kynurenine (Kyn) measurements}

Trp and Kyn concentrations were tested by highperformance liquid chromatography (HPLC) on reversed phase (ProStar 210 - Varian, Palo Alto, CA, USA), according to the protocol described earlier $(17,18)$.

\section{Statistical analysis}

For metabolic analysis data were $\log 2$ transformed to meet assumptions of statistical tests analysis of variance (ANOVA) and post-hoc $t$-tests were performed to identify significant metabolite alterations between the different patient groups and healthy controls. Descriptive analysis was performed for all baseline and demographic characteristics. MannWhitney- $\mathrm{U}$ test was used to compare serum concentrations. 
Table 1 Baseline characteristics of the evaluated cohort

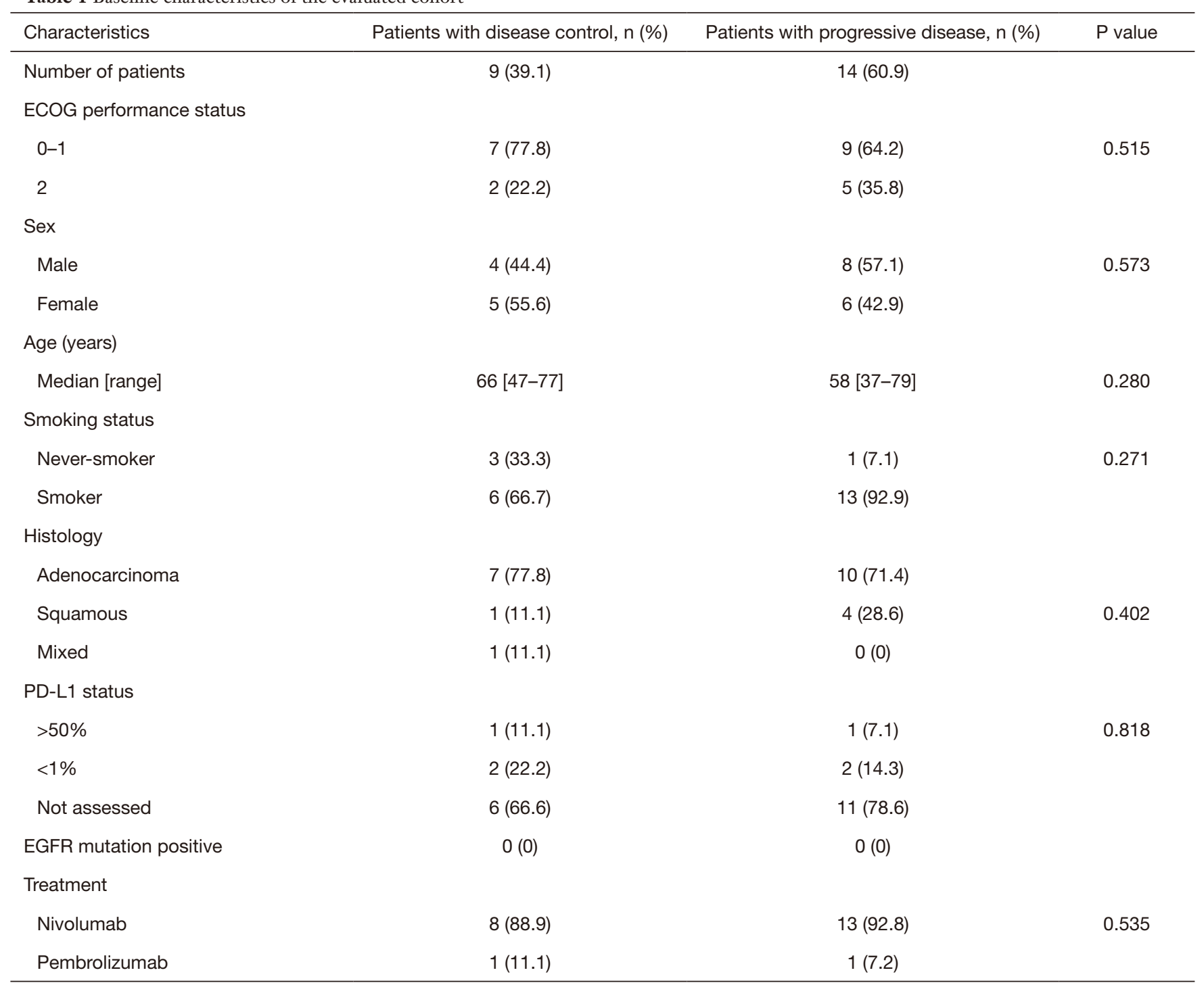

ECOG, Eastern Cooperative Oncology Group; EGFR, epidermal growth factor receptor; PD-L1, programmed death protein-ligand 1.

PFS was defined as time between start of treatment until disease progression or death and was plotted as a curve using the Kaplan-Meier method. Log-rank test was employed for comparison.

Receiver operating characteristics (ROC) analysis was performed to evaluate the most appropriate discriminative threshold of Trp concentrations. A two-tailed $\mathrm{P}$ value $\leq 0.05$ was considered to be statistically significant. SPSS Version 24 (IBM, Armonk, NY, USA) and statistic platform R (Version 3.2.4) were used for statistical analysis.

\section{Results}

\section{Patient characteristics}

Patients' baseline and treatment characteristics are displayed in Table 1. Twenty-three patients with metastatic NSCLC [52.2\% male $(\mathrm{n}=12), 47.8 \%$ female $(\mathrm{n}=11)]$ who were treated in the first-line with a platinum-doublet were included. Patients were treated with nivolumab $(91.3 \%$, $\mathrm{n}=21)$ or pembrolizumab $(8.7 \%, \mathrm{n}=2)$ in the second-line setting. Median follow-up duration of the cohort was 


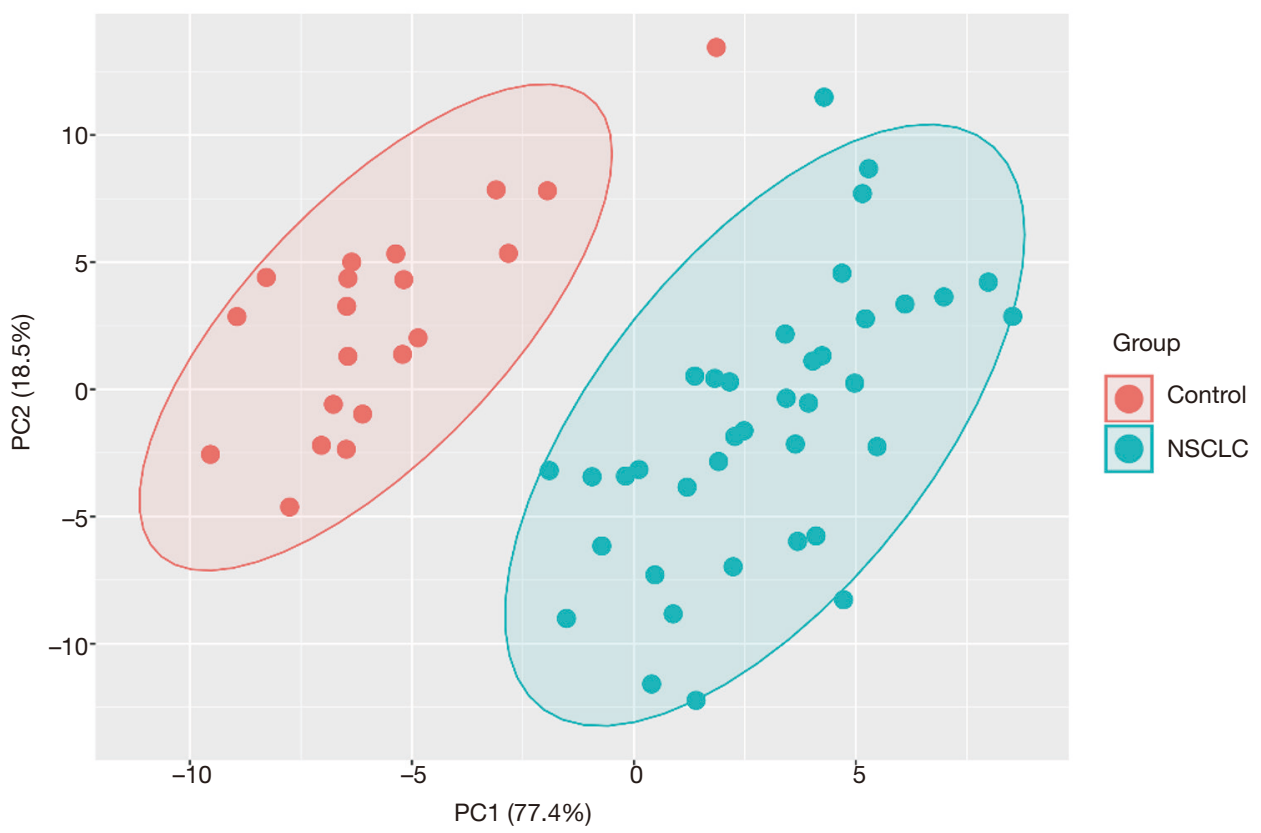

Figure 1 PLS-DA blot of significant metabolic differences between non-small cell lung cancer (NSCLC) patients and healthy controls. PLS-DA, partial least squares discriminant analysis.

327 days (range, 34-668 days). At the first follow up scan, 3 patients showed a partial response (PR; $13.0 \%$ ) and six patients had a stable disease (SD; $26.1 \%$ ) according to iRECIST criteria. Progressive disease (PD) at the first follow up scan was observed in 14 patients $(60.9 \%)$.

\section{The metabolic profile of NSCLC patients differs from bealthy controls}

Plasma samples of 20 patients before start of immunotherapy and at time point of the first follow up scan were available for metabolic analyses. To delineate potential NSCLC-induced metabolic reprogramming we compared the metabolic profile (in total 167 metabolites) of the NSCLC patients with a healthy control group $(n=20)$. In line with already published data (19) we showed that NSCLC patients have a distinct plasma metabolic profile compared to healthy controls (Figure 1). Further analysis yielded significant alterations of 67 metabolites (Figure 2). Concentrations of the amino acids serotonin, taurine, glutamate, arginine, lysine, histidine, sarcosine, leucine, methionine, isoleucine and asparagine were significantly lower in NSCLC patients at baseline compared to healthy controls. Additionally a number of phosphatidylcholines (C34.2, C32.3, C38.0, C36.6, C38.6,
C44.3, C38.1, C36.5, C36.63, C40.1) were significantly decreased, whereas other phosphatidylcholines (C42.5, C44.6, C44.4, C30.0, C44.5, C30.1, C24.0) were upregulated in NSCLC patients. Significantly higher concentrations of sphingomyelins (C16.1, C24.3, C16.0, C22.2, C18.1, C26.1, C24.1, C16.1, C18.0) were observed in the NSCLC group.

\section{Metabolic profile of primary CI resistant NSCLC patients}

Next, we analyzed the metabolic profile of NSCLC patients before start of CI therapy and at the time point of the first follow up scan. The metabolic profile of all analyzed metabolites in our profile was homogenous and could not discriminate between the patients responding or progressing on CI already at the time point of treatment initiation (Figure 3). However, when comparing metabolites at cancer progression with metabolite abundancies at baseline, indoleamine-2,3-dioxygenase (IDO) was significantly upregulated next to phosphatidylcholines (C38.0, C42.6, C36.6), lyso-phosphatidylcholines and total amino acids, whereas branched-chain amino acids (BCAA), including valine, leucine and isoleucine and acylcarnitine $\mathrm{C} 3$ were decreased (Figure 4). Patients responding to therapy showed higher levels of histidine and phenylalanine, whereas 


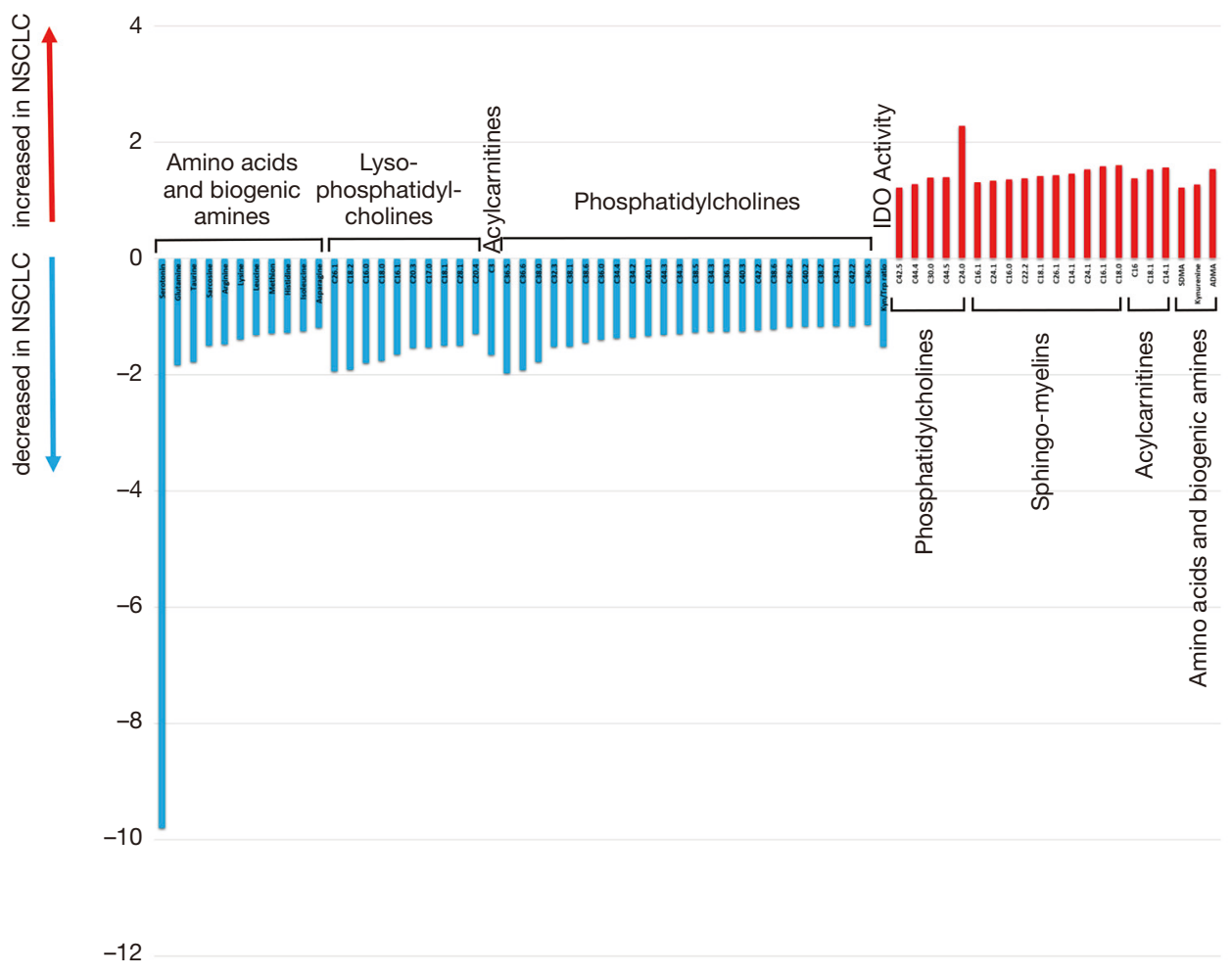

Figure 2 Metabolites significantly altered in patients with NSCLC before treatment start and healthy controls. Fold-changes express the ratio of mean levels of a certain metabolite in the cohort of NSCLC patients divided by the mean level of the same metabolite in the healthy controls. NSCLC, non-small cell lung cancer.

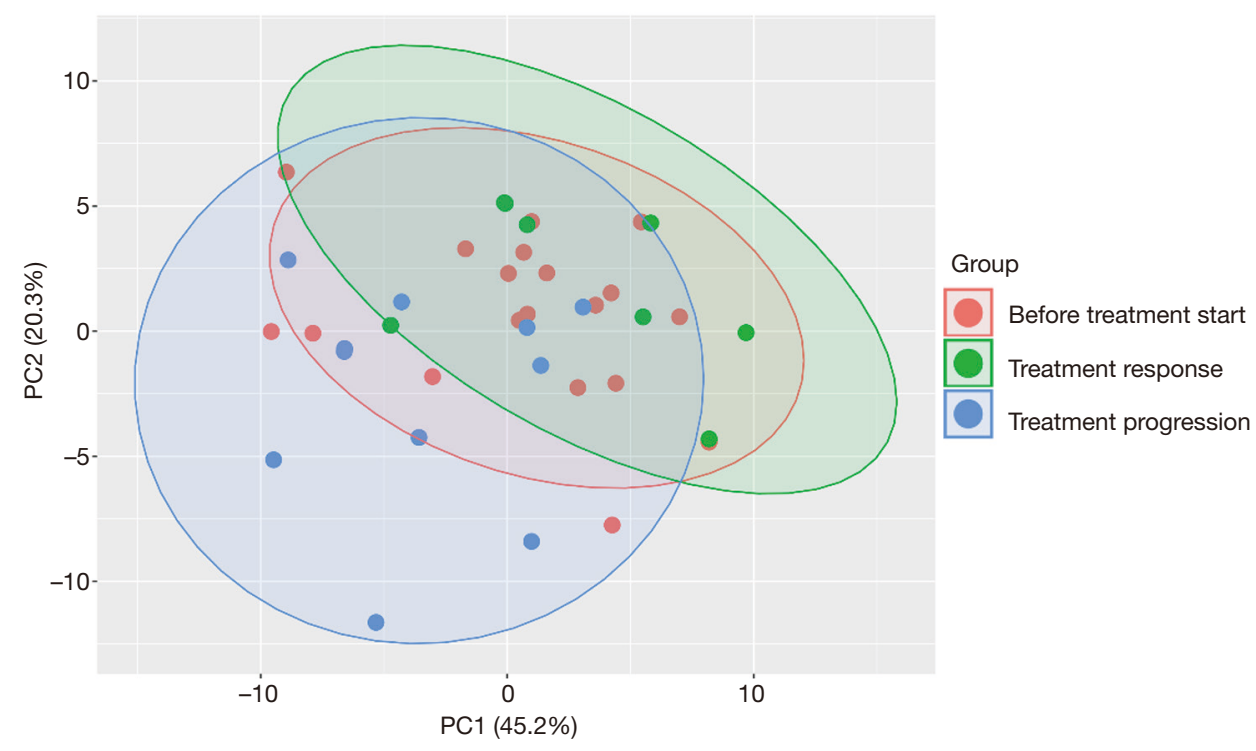

Figure 3 PLS-DA blot of NSCLC patients. Red: before treatment start; blue: progressing on treatment and green: responding on treatment. PLS-DA, partial least squares discriminant analysis. 


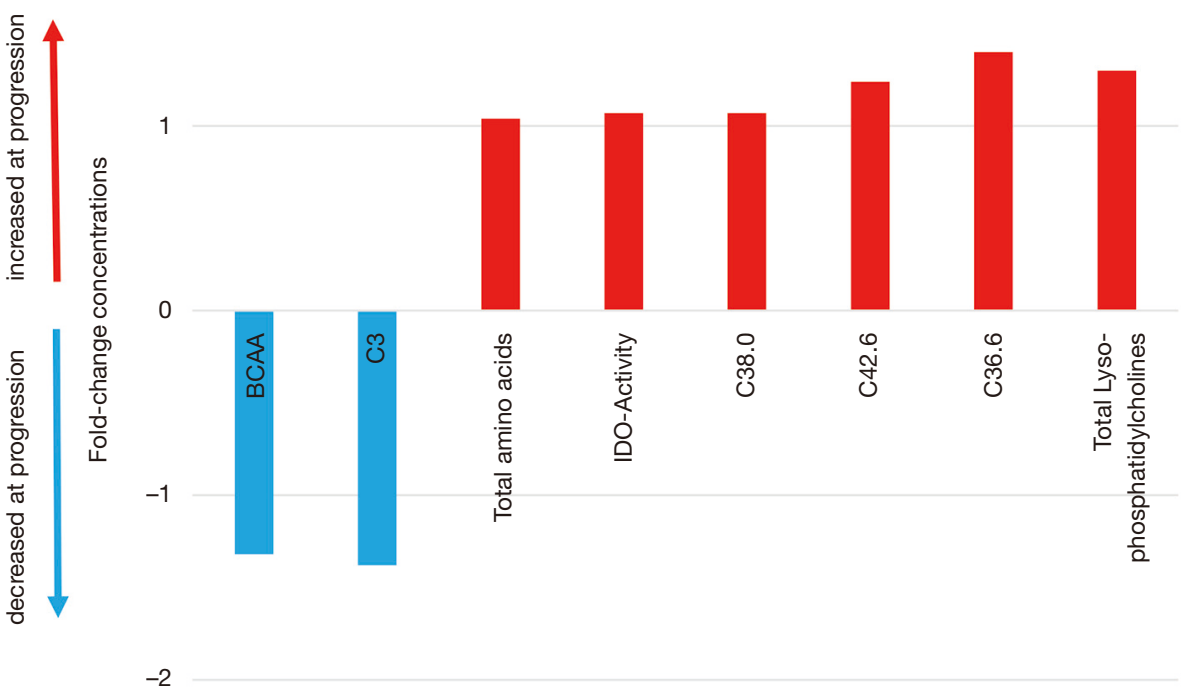

Figure 4 Metabolites significantly altered in patients with NSCLC before treatment start and progressing after the first follow up scan. Fold-changes express the ratio of a certain metabolite in the subcohort of primary refractory NSCLC patients at timepoint of cancer progression divided by baseline levels. NSCLC, non-small cell lung cancer.

glutamate levels were decreased compared to baseline levels. No significant alterations in important metabolites (including IDO and BCAA) were observed in this subset of patients.

\section{Validation experiments of IDO metabolites in NSCLC patients undergoing CI treatment}

To better characterize the metabolic activity of IDO, we next sequentially assessed Kyn and Trp levels by means of HPLC. In the whole cohort of 23 patients median Trp levels, Kyn levels as well as Kyn/Trp were comparable at baseline and at the first follow up scan. Similarly subgroup analysis according to therapeutic response (i.e., controlled disease and primary refractory) showed comparable levels of Trp, Kyn and Kyn/Trp between baseline and first follow up. Most importantly, when comparing baseline Trp levels in patients with controlled disease and primary refractory disease it revealed that patients achieving disease control are characterized by significantly higher Trp levels (median 63.8 vs. $45.0 \mu \mathrm{mol} / \mathrm{L}, \mathrm{P}=0.007$; Figure 5 A). With regard to Kyn and Kyn/Trp no significant differences were observed.

We performed an exploratory ROC analysis to predict disease control upon the follow up scan. A cut-off value above $49.3 \mu \mathrm{mol} / \mathrm{L}$ Trp (AUC 0.833; $\mathrm{P}=0.008$ ) predicted disease control with a sensitivity of $88.89 \%$ (95\% CI: $51.75-99.72 \%)$ and a specificity of $71.43 \%$ (95\% CI: 41.90-91.61\%). Median PFS for all patients was 113 days. Applying the calculated cut-off value according to ROC analysis, median PFS was 61 days in Trp low and 164 days in the Trp high group [HR 0.47 (0.19-1.18), $\mathrm{P}=0.095$; Figure 5B], respectively.

\section{Discussion}

Metabolomics has the potential to discover a multitude of changes that reflect alterations of distinct biological states. In the present study we aimed to investigate the metabolic profile in NSCLC and to detect new biomarkers for response to CI therapy. Our study revealed that 67 out of 167 evaluated metabolites in plasma samples of NSCLC patients are significantly altered compared to healthy controls. These metabolic alterations may be representative of cancer specific intrinsic features and may even be indicative for tumor pathogenesis, which requires a change of blood metabolites that are biologically relevant to sustain survival of cancer cells (20).

In a next step we analyzed metabolic changes during the course of treatment with CI. Therefore, we compared metabolite levels in NSCLC patients with a PD with their 
A

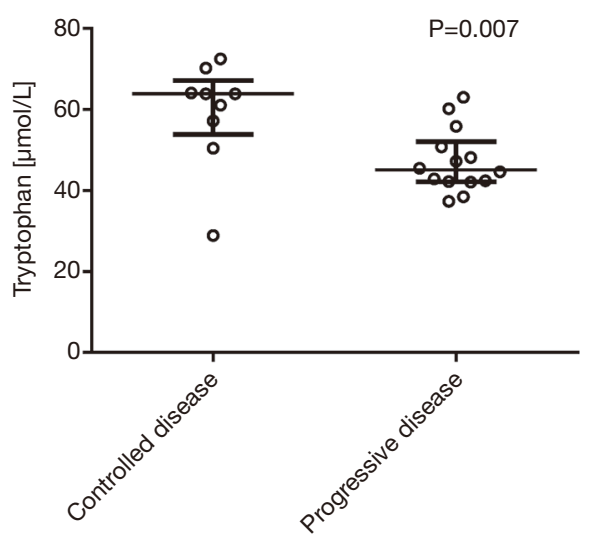

B

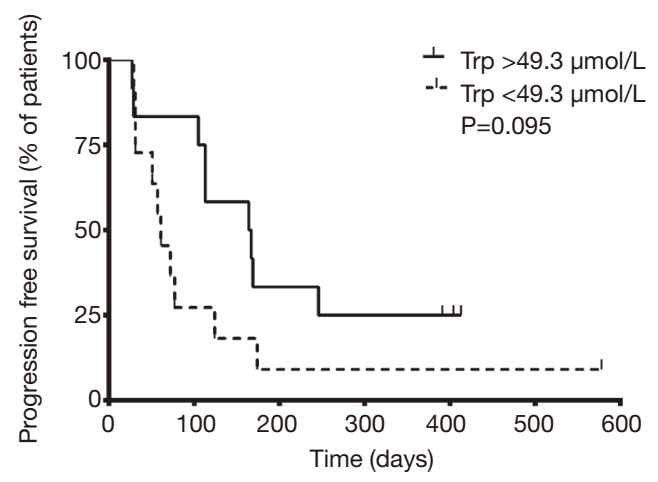

Figure 5 Tryptophan (Trp) levels before treatment start. (A) Median baseline Trp levels in patients with controlled disease (partial response and stable disease) compared to patients with primary refractory disease (median Trp levels controlled disease vs. primary refractory $63.8 v s$. $45.0 \mu \mathrm{mol} / \mathrm{L}, \mathrm{P}=0.007)$. (B) Kaplan-Meier plot showing progression free survival in the whole cohort with stratification according to Trp levels at baseline (the cut-off at Trp $49.3 \mu \mathrm{mol} / \mathrm{L}$ was established according to ROC analysis). A trend towards improved progression-free survival (PFS) was observed in patients with Trp levels $\geq 49.3 \mu \mathrm{mol} / \mathrm{L}$ [PFS $\geq 49.3$ vs. $<49.3 \mu \mathrm{mol} / \mathrm{L}: 164$ vs. 61 days (HR 0.47; 0.19-1.18), P=0.095].

metabolic profile at baseline. We observed that BCAA next IDO metabolites are significantly altered at disease progression. However, these metabolites were not altered in the controlled group, including patients with partial remission or stable disease at first re-evaluation. This finding indicates that metabolic rewiring during course of disease might be an exit strategy in NSCLC to achieve therapeutic resistance upon CI.

BCAA belong to essential amino acids and play a role in weight loss and sarcopenia in cancer patients. NSCLC is characterized by increased BCAA uptake compared to normal lung tissue (21). In vitro and in vivo experiments showed that BCAA are an essential nitrogen source in NSCLC (21). In line with these data we found significantly lower BCAA levels in NSCLC patients compared to healthy controls. Furthermore, the data by Mayers et al. showed that BCAA levels decreased in patients progressing under treatment (21).

The TME is critical for cancer immune surveillance and immune tolerance, in which Trp catabolism plays a key role. Trp is an essential amino acid for innate immune cells and its breakdown by the enzyme IDO to Kyn has been found to be of vast importance in tolerogenic processes (22). According to literature (23-25), IDO is induced by proinflammatory signals, mainly by type- 1 interferons, and is expressed by malignant cells, stromal cells, lymphocytes and dendritic cells. Local Trp deficiency and higher Kyn concentrations lead to T-cell anergy, as well as Treg differentiation and activation. Moreover, high
IDO activity in cancer patients was associated with an unfavorable prognosis and a more aggressive disease (26-30). Since IDO metabolism was one of the most up-regulated metabolic pathways in PD we set out to validate this finding with a second method. We showed that baseline serum Trp levels were significantly higher in patients with controlled disease upon CI therapy.

Similar to our observations Botticelli and colleagues showed that baseline Kyn/Trp correlated inversely with PFS (HR 0.3; $\mathrm{P}=0.018$ ) as well as OS (HR 0.3; $\mathrm{P}=0.0005$ ) in NSCLC patients undergoing CI (31). In a study investigating IDO in patients with early stage NSCLC undergoing curative radiotherapy, higher Kyn levels after radiotherapy were associated with poor outcome (32). In our study the Kyn/Trp failed to predict response and was not associated with a significant difference in PFS. Even though increased IDO activity should be reflected by Kyn/ Trp, Trp was the only predictive marker in our study cohort. We assume that a significant difference might be concealed due to the small sample size in our study.

Interestingly, Trp levels of patients with cancer progression are comparable to previously published values of healthy controls. A higher IDO activity and therefore lower Trp concentration as compared to healthy controls has been described in various studies (29,33-35). This might reflect an increased immunogenic tumor state in patients with a controlled disease upon CI. In line with this data $\mathrm{Li}$ et al. could show that an increase of the Kyn/Trp during therapy is associated with a secondary resistance towards CI 
with a consequently bad outcome (36).

We performed a ROC analysis of baseline Trp levels to evaluate its predictive value with regards to response upon the first follow up scan. In this model, a cut-off $\geq 49.3 \mu \mathrm{mol} / \mathrm{L}$ predicted disease control with a sensitivity of approximately $89 \%$. To the best of our knowledge there is no direct comparison of PD-L1 and IDO activity as predictive biomarkers in NSCLC patients. We speculate that measurement of IDO activity, reflected by Trp baseline levels, might be an additional predictive biomarker in CI therapy. Further validation and direct comparison are desirable.

Although this promising data, we have several limitations. Data coming from these analyses were of retrospective nature and were performed in a single center with a limited small sample size. Also, at the starting of sampling and experiments, CIs had been approved only recently and were therefore only used in patients in the second-line. Today, CIs are used in first-line setting for metastatic NSCLC.

IDO metabolism is an attractive treatment target due to its importance in immunogenic pathways and the TME. However, while an early trial showed encouraging results, a latter phase III trial did not meet the expectations. In the phase I/II trial ECHO-202/Keynote-037 yielded promising responses in patients with solid tumors (including NSCLC) when using the oral IDO inhibitor epacadostat (37). The phase 3 ECHO-301/KEYNOTE-2528 trial on the other hand did not meet its primary endpoint to improve PFS in patients treated with epacadostat in combination with pembrolizumab (38). The lack of efficacy is believed to be caused amongst others by the study design (38) and an unsatisfactory pharmacokinetic profile of epacadostat (39). Moreover, to the best of our knowledge, in none of these trials the expression of IDO was assessed before treatment start, neither in the peripheral blood nor by immunohistochemistry. We found, that patients with a lower Trp concentration, reflecting higher IDO activity, were more likely to progress under CI. Therefore, we hypothesize that patients with high IDO activity, which leads to a more tolerogenic state in the TME by upregulating Treg cells and downregulating CD8 and CD4 T-cells $(23,24)$ may profit from an IDO inhibitor to enhance the activity of immunogenic cells. Thus, in NSCLC patients with low Trp concentrations at baseline the combination of IDO-inhibitors and CIs might be beneficial to increase response. This could possibly turn an immunosenescent tumor into an immunogenic state, leading to an increased efficacy of checkpoint inhibition and might overcome resistance to CIs.

\section{Conclusions}

NSCLC is characterized by metabolic alterations compared to healthy controls. Furthermore we show that metabolic rewiring is associated with CI resistance. IDO metabolism seems to be up-regulated in primary CI resistance. Therefore, Trp levels could be used as surrogate parameter of IDO activity and may serve as a rational biomarker to select for patients qualifying for a combined IDO inhibitor ICI therapy within future clinical trials.

\section{Acknowledgments}

Funding: The study was supported by the Verein fuer tumorforschung (Innsbruck).

\section{Footnote}

Reporting Checklist: The authors have completed the STARD reporting checklist. Available at http://dx.doi.org/10.21037/ tlcr-20-380

Data Sharing Statement: Available at http://dx.doi. org/10.21037/tlcr-20-380

Peer Review File: Available at http://dx.doi.org/10.21037/ tlcr-20-380

Conflicts of Interest: All authors have completed the ICMJE uniform disclosure form (available at http://dx.doi. org/10.21037/tlcr-20-380). The authors have no conflicts of interest to declare.

Etbical Statement: The authors are accountable for all aspects of the work in ensuring that questions related to the accuracy or integrity of any part of the work are appropriately investigated and resolved. The study has conformed to the provisions of the Declaration of Helsinki (as revised in 2013). The study was approved by the local ethic committee of the Medical University of Innsbruck (local ethics committee study number: AN2015-0017 $345 / 4.20$ ) and informed consent was taken from all the patients.

Open Access Statement: This is an Open Access article distributed in accordance with the Creative Commons 
Attribution-NonCommercial-NoDerivs 4.0 International License (CC BY-NC-ND 4.0), which permits the noncommercial replication and distribution of the article with the strict proviso that no changes or edits are made and the original work is properly cited (including links to both the formal publication through the relevant DOI and the license). See: https://creativecommons.org/licenses/by-nc-nd/4.0/.

\section{References}

1. Siegel RL, Miller KD, Jemal A. Cancer statistics, 2020. CA Cancer J Clin 2020;70:7-30.

2. Brahmer J, Reckamp KL, Baas P, et al. Nivolumab versus Docetaxel in Advanced Squamous-Cell Non-Small-Cell Lung Cancer. N Engl J Med 2015;373:123-35.

3. Borghaei H, Paz-Ares L, Horn L, et al. Nivolumab versus Docetaxel in Advanced Nonsquamous Non-Small-Cell Lung Cancer. N Engl J Med 2015;373:1627-39.

4. Garon EB, Rizvi NA, Hui R, et al. Pembrolizumab for the treatment of non-small-cell lung cancer. N Engl J Med 2015;372:2018-28.

5. Gettinger SN, Horn L, Gandhi L, et al. Overall Survival and Long-Term Safety of Nivolumab (Anti-Programmed Death 1 Antibody, BMS-936558, ONO-4538) in Patients With Previously Treated Advanced Non-Small-Cell Lung Cancer. J Clin Oncol 2015;33:2004-12.

6. Cairns RA, Harris IS, Mak TW. Regulation of cancer cell metabolism. Nat Rev Cancer 2011;11:85-95.

7. DeBerardinis RJ, Chandel NS. Fundamentals of cancer metabolism. Sci Adv 2016;2:e1600200.

8. Cantor JR, Sabatini DM. Cancer cell metabolism: one hallmark, many faces. Cancer Discov 2012;2:881-98.

9. Martín-Martín N, Carracedo A, Torrano V. Metabolism and Transcription in Cancer: Merging Two Classic Tales. Front Cell Dev Biol 2017;5:119.

10. Pavlova NN, Thompson CB. The Emerging Hallmarks of Cancer Metabolism. Cell Metab 2016;23:27-47.

11. Hanahan D, Weinberg RA. Hallmarks of cancer: the next generation. Cell 2011;144:646-74.

12. Matassa DS, Amoroso MR, Lu H, et al. Oxidative metabolism drives inflammation-induced platinum resistance in human ovarian cancer. Cell Death Differ 2016;23:1542-54.

13. Denise C, Paoli P, Calvani M, et al. 5-fluorouracil resistant colon cancer cells are addicted to OXPHOS to survive and enhance stem-like traits. Oncotarget 2015;6:41706-21.

14. Wang Z, Yip LY, Lee JHJ, et al. Methionine is a metabolic dependency of tumor-initiating cells. Nat Med
2019;25:825-37.

15. Skoulidis F, Goldberg ME, Greenawalt DM, et al. Mutations and PD-1 Inhibitor Resistance in. Cancer Discov 2018;8:822-35.

16. Najjar YG, Menk AV, Sander C, et al. Tumor cell oxidative metabolism as a barrier to PD-1 blockade immunotherapy in melanoma. JCI Insight 2019;4:e124989.

17. Laich A, Neurauter G, Widner B, et al. More rapid method for simultaneous measurement of tryptophan and kynurenine by HPLC. Clin Chem 2002;48:579-81.

18. Widner B, Werner ER, Schennach H, et al. Simultaneous measurement of serum tryptophan and kynurenine by HPLC. Clin Chem 1997;43:2424-6.

19. Xu S, Zhou Y, Geng H, et al. Serum Metabolic Profile Alteration Reveals Response to Platinum-Based Combination Chemotherapy for Lung Cancer: Sensitive Patients Distinguished from Insensitive ones. Sci Rep 2017;7:17524.

20. Griffin JL, Shockcor JP. Metabolic profiles of cancer cells. Nat Rev Cancer 2004;4:551-61.

21. Mayers JR, Torrence ME, Danai LV, et al. Tissue of origin dictates branched-chain amino acid metabolism in mutant Kras-driven cancers. Science 2016;353:1161-5.

22. Moffett JR, Namboodiri MA. Tryptophan and the immune response. Immunol Cell Biol 2003;81:247-65.

23. Munn DH, Sharma MD, Baban B, et al. GCN2 kinase in $\mathrm{T}$ cells mediates proliferative arrest and anergy induction in response to indoleamine 2,3-dioxygenase. Immunity 2005;22:633-42.

24. Fallarino F, Grohmann U, You S, et al. The combined effects of tryptophan starvation and tryptophan catabolites down-regulate $T$ cell receptor zeta-chain and induce a regulatory phenotype in naive $\mathrm{T}$ cells. J Immunol 2006;176:6752-61.

25. Munn DH, Mellor AL. IDO in the Tumor Microenvironment: Inflammation, Counter-Regulation, and Tolerance. Trends Immunol 2016;37:193-207.

26. Godin-Ethier J, Hanafi LA, Piccirillo CA, et al. Indoleamine 2,3-dioxygenase expression in human cancers: clinical and immunologic perspectives. Clin Cancer Res 2011;17:6985-91.

27. Huang A, Fuchs D, Widner B, et al. Serum tryptophan decrease correlates with immune activation and impaired quality of life in colorectal cancer. Br J Cancer 2002;86:1691-6.

28. Weinlich G, Murr C, Richardsen L, et al. Decreased serum tryptophan concentration predicts poor prognosis in malignant melanoma patients. Dermatology 
2007;214:8-14.

29. Brandacher G, Winkler C, Schroecksnadel K, et al. Antitumoral activity of interferon-gamma involved in impaired immune function in cancer patients. Curr Drug Metab 2006;7:599-612.

30. Seeber A, Klinglmair G, Fritz J, et al. High IDO-1 expression in tumor endothelial cells is associated with response to immunotherapy in metastatic renal cell carcinoma. Cancer Sci 2018;109:1583-91.

31. Botticelli A, Cerbelli B, Lionetto L, et al. Can IDO activity predict primary resistance to anti-PD-1 treatment in NSCLC? J Transl Med 2018;16:219.

32. Wang W, Huang L, Jin JY, et al. A Validation Study on IDO Immune Biomarkers for Survival Prediction in NonSmall Cell Lung Cancer: Radiation Dose Fractionation Effect in Early-Stage Disease. Clin Cancer Res 2020;26:282-9.

33. Engin AB, Ozkan Y, Fuchs D, et al. Increased tryptophan degradation in patients with bronchus carcinoma. Eur J Cancer Care (Engl) 2010;19:803-8.

34. Suzuki Y, Suda T, Furuhashi K, et al. Increased serum kynurenine/tryptophan ratio correlates with disease

Cite this article as: Kocher F, Amann A, Zimmer K, Geisler S, Fuchs D, Pichler R, Wolf D, Kurz K, Seeber A, Pircher A. High indoleamine-2,3-dioxygenase 1 (IDO) activity is linked to primary resistance to immunotherapy in non-small cell lung cancer (NSCLC). Transl Lung Cancer Res 2021;10(1):304-313. doi: 10.21037/tlcr-20-380 progression in lung cancer. Lung Cancer 2010;67:361-5.

35. Sagan D, Kocki T, Kocki J, et al. Serum kynurenic acid: possible association with invasiveness of non-small cell lung cancer. Asian Pac J Cancer Prev 2012;13:4241-4.

36. Li H, Bullock K, Gurjao C, et al. Metabolomic adaptations and correlates of survival to immune checkpoint blockade. Nat Commun 2019;10:4346.

37. Mitchell TC, Hamid O, Smith DC, et al. Epacadostat Plus Pembrolizumab in Patients With Advanced Solid Tumors: Phase I Results From a Multicenter, Open-Label Phase I/II Trial (ECHO-202/KEYNOTE-037). J Clin Oncol 2018;36:3223-30.

38. Long G, Dummer R, Hamid O, et al. Epacadostat plus pembrolizumab versus placebo plus pembrolizumab in patients with unresectable or metastatic melanoma (ECHO-301/KEYNOTE-252): a phase 3, randomised, double-blind study. Lancet Oncol 2019;20:1083-97.

39. Song X, Sun P, Wang J, et al. Design, synthesis, and biological evaluation of 1,2,5-oxadiazole-3carboximidamide derivatives as novel indoleamine2,3-dioxygenase 1 inhibitors. Eur J Med Chem 2020;189:112059. 